University of Nebraska - Lincoln

DigitalCommons@University of Nebraska - Lincoln

Publications from USDA-ARS / UNL Faculty

U.S. Department of Agriculture: Agricultural

Research Service, Lincoln, Nebraska

$5-15-2017$

\title{
Morphological and molecular characterization of Sarcocystis arctica-like sarcocysts from the Arctic fox (Vulpes lagopus) from Alaska, USA
}

\author{
Camila K. Cerqueira-Cézar \\ United States Department of Agriculture, Beltsville Agricultural Research Center, Building 1001, Beltsville \\ Peter C. Thompson \\ United States Department of Agriculture, Beltsville Agricultural Research Center, Building 1001, Beltsville \\ Shiv Kummar Verma \\ United States Department of Agriculture, Beltsville Agricultural Research Center, Building 1001, Beltsville \\ Joseph Mowery \\ Beltsville Agricultural Research Center, Building 12, Beltsville \\ Rafael Calero-Bernal \\ United States Department of Agriculture, Beltsville Agricultural Research Center, Building 1001, Beltsville
}

See next page for additional authors

Follow this and additional works at: https://digitalcommons.unl.edu/usdaarsfacpub

Cerqueira-Cézar, Camila K.; Thompson, Peter C.; Verma, Shiv Kummar; Mowery, Joseph; Calero-Bernal, Rafael; Antunes Murata, Fernando H.; Sinnett, David R.; Hemert, Caroline Van; Rosenthal, Benjamin M.; and Dubey, Jitender P., "Morphological and molecular characterization of Sarcocystis arctica-like sarcocysts from the Arctic fox (Vulpes lagopus) from Alaska, USA" (2017). Publications from USDA-ARS / UNL Faculty. 2230.

https://digitalcommons.unl.edu/usdaarsfacpub/2230

This Article is brought to you for free and open access by the U.S. Department of Agriculture: Agricultural Research Service, Lincoln, Nebraska at DigitalCommons@University of Nebraska - Lincoln. It has been accepted for inclusion in Publications from USDA-ARS / UNL Faculty by an authorized administrator of DigitalCommons@University of Nebraska - Lincoln. 


\section{Authors}

Camila K. Cerqueira-Cézar, Peter C. Thompson, Shiv Kummar Verma, Joseph Mowery, Rafael Calero-

Bernal, Fernando H. Antunes Murata, David R. Sinnett, Caroline Van Hemert, Benjamin M. Rosenthal, and Jitender P. Dubey 


\title{
Morphological and molecular characterization of Sarcocystis arctica-like sarcocysts from the Arctic fox (Vulpes lagopus) from Alaska, USA
}

\author{
Camila K. Cerqueira-Cézar ${ }^{1}$ - Peter C. Thompson ${ }^{1} \cdot$ Shiv Kumar Verma ${ }^{1}$. \\ Joseph Mowery $^{2} \cdot$ Rafael Calero-Bernal $^{1} \cdot$ Fernando H. Antunes Murata $^{1}$. \\ David R. Sinnett ${ }^{3}$ - Caroline Van Hemert ${ }^{4} \cdot$ Benjamin M. Rosenthal $^{1} \cdot$ Jitender P. Dubey $^{1}$
}

\begin{abstract}
The muscles of herbivores commonly harbor sarcocysts of parasites belonging to species in the genus Sarcocystis, but such muscle parasites are rare in carnivores. Here, we report Sarcocystis arctica-like sarcocysts in muscles of Arctic foxes (Vulpes lagopus) from Alaska, USA, for the first time. The tongues of 56 foxes were examined for Sarcocystis infection using several methods. Sarcocystis bradyzoites were detected in pepsin digests of 13 (23.2\%), and sarcocysts were found in histological sections stained with hematoxylin and eosin (HE) of 9 (16.0\%). By light microscopy, sarcocysts were up to $4 \mathrm{~mm}$ long and up to $245 \mu \mathrm{m}$ wide. In HE-stained sections, the sarcocyst wall appeared smooth and up to $1.5 \mu \mathrm{m}$ thick without visible protrusions. By transmission electron microscopy, the sarcocyst wall had a wavy parasitophorous vacuolar membrane (pvm) folded as pleomorphic villar protrusions (vp), sometimes with anastomoses of villar tips. The $\mathrm{vp}$ and the ground substance (gs) layer were smooth and without microtubules. The gs was up to $2.0 \mu \mathrm{m}$
\end{abstract}

Jitender P. Dubey

Jitender.Dubey@ars.usda.gov

1 Agricultural Research Service, Animal Parasitic Diseases Laboratory, United States Department of Agriculture, Beltsville Agricultural Research Center, Building 1001, Beltsville, MD 20705-2350, USA

2 Agricultural Research Service, Electron and Confocal Microscopy Unit, United States Department of Agriculture, Beltsville Agricultural Research Center, Building 12, Beltsville, MD 20705-2350, USA

3 Animal and Plant Health Inspection Service, Wildlife Services, National Wildlife Disease Program, United States Department of Agriculture, 9001 E. Frontage Rd, Palmer, AK 99645, USA

4 United States Geological Survey, Alaska Science Center, 4210 University Drive, Anchorage, AK 99508, USA thick. The total width of the wall including vp and the gs was up to $4.0 \mu \mathrm{m}$. The vp were up to $3.0 \mu \mathrm{m}$ long and most closely resembled "type 9c." All sarcocysts were mature and contained numerous $8.1 \times 2.1 \mu \mathrm{m}$ sized bradyzoites. Molecular characterization (at 18S rDNA, 28S rDNA, ITS1 , and cox1) showed the highest affinity for $S$. arctica of the Arctic fox (V. lagopus) from Norway. In the present investigation, we provide evidence that sarcocysts are common in tongues of Alaskan Arctic foxes suggesting that these carnivores are serving as intermediate hosts, and we also provide ultrastructure of $S$. arctica from the Arctic fox for the first time.

Keywords Arctic fox $\cdot$ Vulpes lagopus $\cdot$ Sarcocystis · Ultrastructure $\cdot$ Phylogeny

\section{Introduction}

Species in the genus Sarcocystis have an obligate twohost life cycle, with sexual development in small intestine of the definitive host and asexual development in the intermediate host, usually herbivores (Dubey et al. 2016). Canids are known definitive hosts for numerous species of Sarcocystis that form sarcocysts in domestic and wild herbivores. Occasionally, muscular sarcocysts have been reported in canids, including dogs, coyotes, wolves, and foxes. The clinical and biologic significance of sarcocysts in canids are unknown, except severe myositis was reported in four dogs in association with two Sarcocystis species (Dubey et al. 2015). Recently, muscular sarcocysts were detected in 19 of $36(52.8 \%)$ Pampas foxes (Lycalopex gymnocercus) from Argentina (Scioscia et al. 2017). The life cycle of species of Sarcocystis that form 
sarcocysts in canid muscle are unknown. It remains uncertain whether canids are aberrant hosts for sarcocysts or if there is a regular cycle entailing transmission through canid muscles. If so, predation or scavenging of canid carcasses by a carnivore would be suspected as necessary.

Sarcocysts were reported in two Arctic foxes from Norway and named Sarcocystis arctica based on light microscopic examination and molecular characterization of frozen sarcocysts; ultrastructure of sarcocysts was not reported (Gjerde and Schulze 2014). Ultrastructure of the sarcocyst wall is an important taxonomic criterion (Dubey et al. 2016). In the present study, we report ultrastructure of fresh $S$. arctica-like sarcocysts from the Arctic fox (Vulpes lagopus) from Alaska, USA, and provide molecular data. Additionally, we report high prevalence of this parasite indicating that Arctic fox is a regular intermediate hosts for this species.

\section{Material and methods}

\section{Sample collection}

Samples of tongue were collected from 56 foxes between May and June 2016 on the Arctic Coastal Plain near Barrow, Alaska $\left(71.29^{\circ} \mathrm{N}, 156.79^{\circ} \mathrm{W}\right)$. All foxes were shot or trapped (ADFG permit 16-121) as part of a predator control program designated under the US Fish and Wildlife Service Steller's Eider (Polysticta stelleri) recovery plan using methods in accordance with guidelines from the American Veterinary Medical Association and the American Society of Mammalogists. The tongues were removed from carcasses using a scalpel, refrigerated, and shipped on ice to the US Department of Agriculture Animal Parasitic Diseases Laboratory in Beltsville, MD within 4 days.

\section{Muscle squashes}

Each tongue sample was examined for the presence of Sarcocystis sarcocysts by squeezing fresh pieces of muscle between the slide and coverslip.

\section{Pepsin digestion}

Most of the remaining tongue samples (10 g) were homogenized in saline and incubated in acid pepsin solution for $1 \mathrm{~h}$ at $37^{\circ} \mathrm{C}$ in a water bath, as described previously (Dubey 2010). After centrifugation at $1180 \mathrm{~g}$ for $10 \mathrm{~min}$, the supernatant was discarded, and the sediment was suspended in $5 \mathrm{ml}$ of saline; a drop $(0.1 \mathrm{ml})$ was spread on a slide, covered with a $22 \times 22 \mathrm{~mm}$ coverslip, and examined microscopically for bradyzoite presence at $\times 200-400$ magnification . Additionally, bradyzoites were smeared on glass slides, air dried, and stained with Giemsa.

\section{Measurements}

Measurements were made digitally using Olympus DP73 camera.

\section{Histological examination}

One piece from the 56 tongues was fixed in $10 \%$ buffered formalin. After fixing, several pieces of tongue were cut to

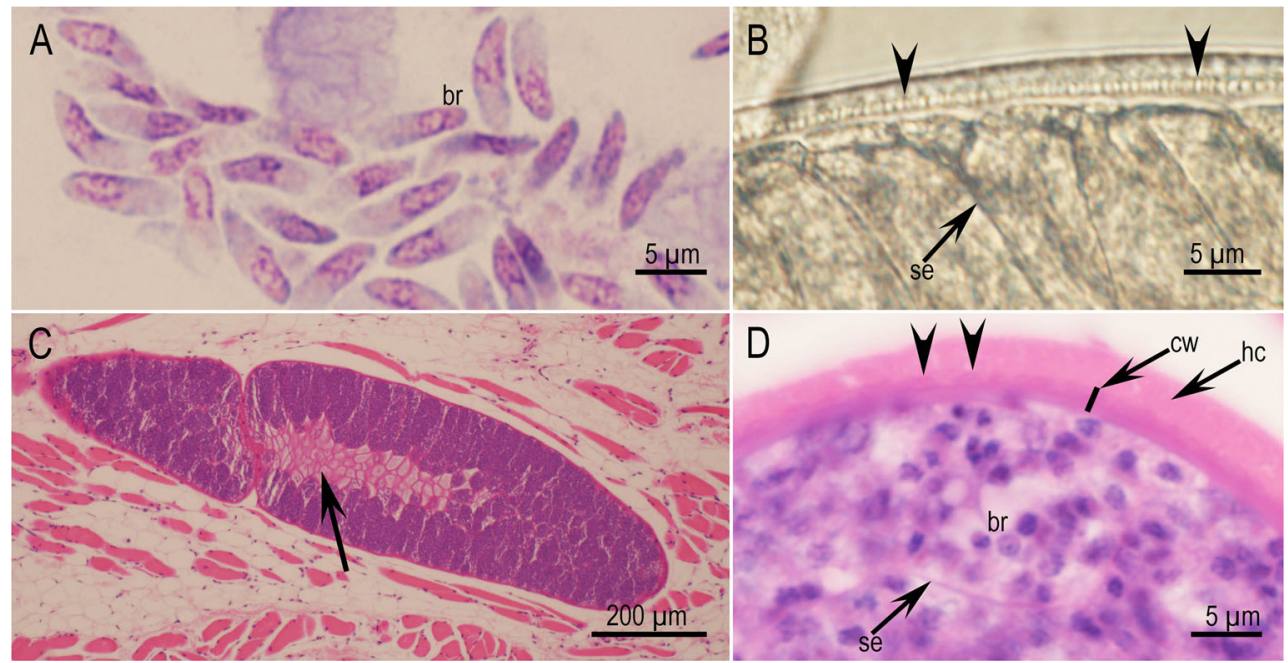

Fig. 1 Sarcocystis arctica-like sarcocysts and bradyzoites from the tongue of the Alaska Arctic foxes. a Bradyzoites in pepsin digest. Smear. Giemsa stain. b Sarcocyst. Unstained Note septa (se) and villar protrusions (arrowheads) on the cyst wall. c Longitudinal section of sarcocyst. Hematoxylin and eosin stain. Note empty space (arrow) in the interior of the sarcocyst. d Part of cross section of sarcocyst showing indistict protrusions and numerous bradyzoites. Note cyst wall $(c w)$ with indistinct protrusions (arrowheads) into the host cell $(h c)$, and numerous bradyzoites $(b r)$. 


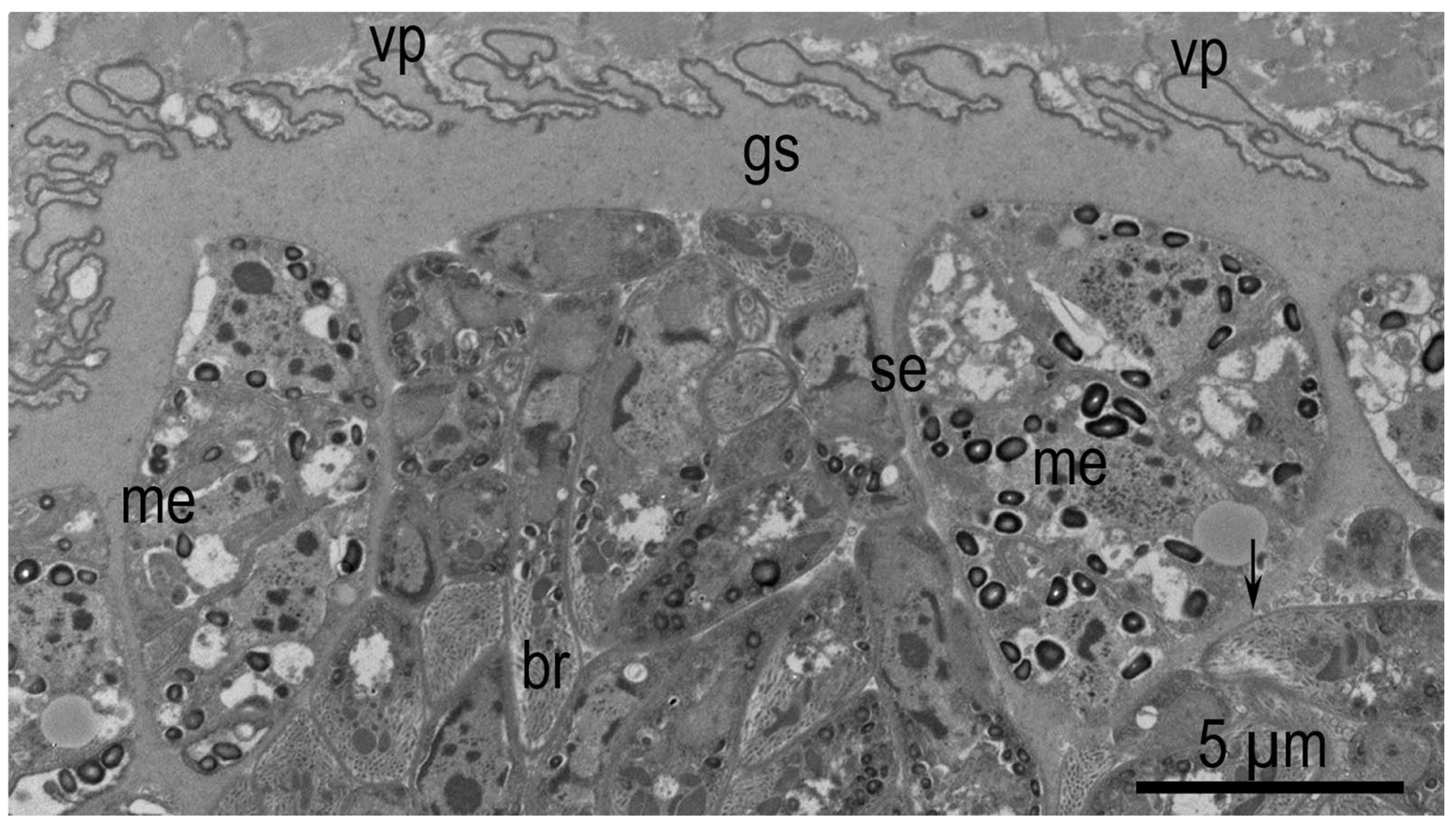

Fig. 2 TEM micrographs of a sarcocyst from the tongue of the Alaskan Arctic fox. Note, pleomorphic villar protrusions ( $v p$ ), thick ground substance $(g s)$, with prominent septa $(s e)$, metrocytes $(m e)$, and bradyzoites $(b r)$.

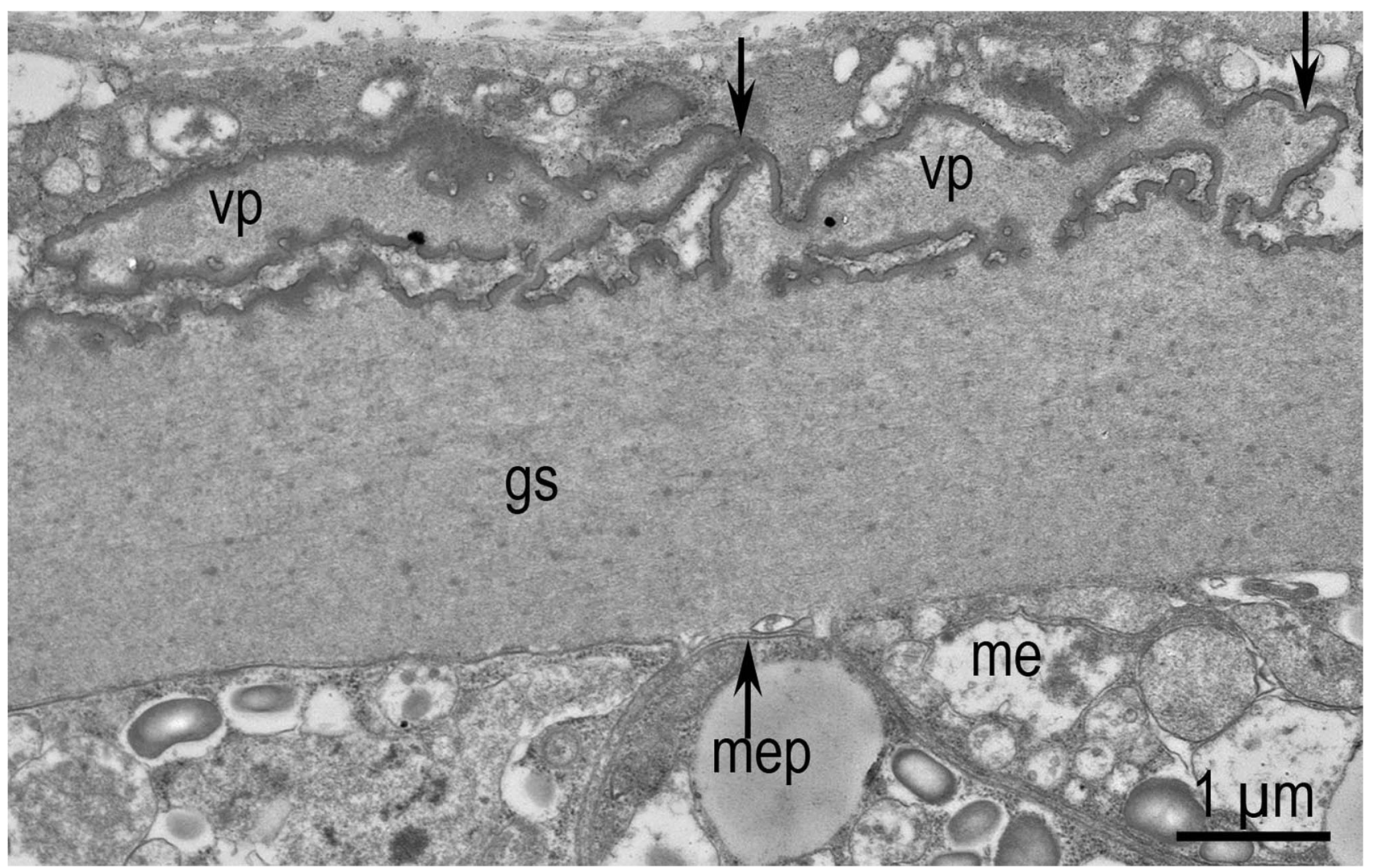

Fig. 3 TEM of sarcocyst \#1 from the Alaskan Arctic fox. Note pleomorhic, anastomosing (arrows) villar protrusions ( $v p)$, metrocytes ( $m e$ ), and ground substance layer $(g s)$ justapoxed with metrocyte pellicle (mep) 
fit a $3 \times 2 \mathrm{~cm}$ paraffin block and were processed for histology. Sections were cut $5 \mu \mathrm{m}$ thick and examined microscopically after staining with hematoxylin and eosin (HE). All sarcocysts in a block were counted.

\section{Transmission electron microscopic examination}

Sarcocysts from two, heavily infected tongues were fixed in glutaraldehyde and processed for transmission electron microscopy (TEM) as described previously (Trupkiewicz et al. 2016).

\section{Molecular characterization}

From four positive animals, five morphologically similar sarcocysts were excised and individually placed in $20 \mu \mathrm{l}$ of saline $(0.85 \%)$ in $1.5 \mathrm{ml}$ centrifuge tubes, which were kept frozen at $-20{ }^{\circ} \mathrm{C}$ until DNA isolation.

DNA was extracted from two sarcocysts from two foxes using DNeasy Blood and Tissue Kit (Qiagen Inc., Valencia, CA, USA), according to manufacturer's instructions. The extracted DNA was characterized by PCR amplification and sequencing of $18 \mathrm{~S}, 28 \mathrm{~S}$, and ITS1 of the ribosomal DNA in the nuclear genome and cytochrome $\mathrm{c}$ oxidase subunit 1 gene (cox 1 ) from the mitochondrial genome using previously published primers (Gjerde 2013, 2014; Gjerde and Josefsen 2015). Each locus was amplified independently using $3 \mu \mathrm{l}$ template, $1 \mu \mathrm{l}$ of forward and reverse primers $(20 \mathrm{mM}$ final concentration), $12.5 \mu \mathrm{l}$ of Taq PCR Master Mix (Qiagen, USA) and $8.5 \mu \mathrm{l}$ of distilled water. Following an initial denaturation step of 5 min at $95^{\circ} \mathrm{C}, 40$ cycles of PCR were conducted for all reactions with the following cycling conditions $\left(95^{\circ} \mathrm{C}\right.$ for $45 \mathrm{~s}, 56.5^{\circ} \mathrm{C}$ for $45 \mathrm{~s}$, and $72^{\circ} \mathrm{C}$ for $1 \mathrm{~min}$ ), ending with $10 \mathrm{~min}$ at $72{ }^{\circ} \mathrm{C}$ for final extension.

After amplification, PCR products were visualized on $2.5 \%$ agarose gels stained with ethidium bromide, and single PCR amplicons were excised and purified using QIAquick Gel Extraction Kits (Qiagen Inc., Valencia, CA, USA) according to manufacturer's instructions. For sequencing the DNA, $1 \mu \mathrm{l}$ of each purified amplicon was sequenced using the original amplification primers and BigDye Terminator v3.1 chemistry according to manufacturer's instructions (Applied Biosystems, Foster City, CA, USA). Sequencing products were purified by EdgeBio Performa DTR Gel Filtration Cartridges (Cat\# 42453) and sequenced on an ABI 3730 Sequencer. Sequences were aligned in Geneious R9 using the MUSCLE plugin and neighbor-joining trees were built with 1000 bootstraps. Similarities between newly generated sequences were compared to those deposited in the NCBI database. All sequences derived from Alaskan Arctic fox have been deposited in GenBank.

\section{Ethics}

All research was performed following the approved protocols by the institutions involved.

\section{Results}

\section{Light microscopy}

In unstained tongue squashes, four (7.1\%) foxes were positive and sarcocysts were slender with an average size of $1960 \mu \mathrm{m}$ (1105-4080, $n=31)$ long and $202 \mu \mathrm{m}(134-288)$ wide. Numerous bradyzoites were detected microscopically in 13 (23.2\%) tongue pepsin digest (Fig. 1a). These bradyzoites measured $8.1 \times 2.1(6.9-9.5 \times 1.4-2.7, n=51) \mu \mathrm{m}$ in size. The sarcocyst wall appeared to have small protrusions when viewed at $\times 1000$ magnification of unstained squashes (Fig. 1b).

Sarcocysts were found in HE-stained sections in nine (16.0\%) foxes (Fig. 1c). The number of sarcocysts per block was low $(<10)$ in six foxes. However, numerous sarcocysts were seen in three foxes with up to 107 sarcocysts in one fox.



Fig. 4 TEM of sarcocyst \#1 from the Alaskan Arctic fox Note host cell $(h c)$, microtubules $(m t)$, and wavy parasitophorous vacuolar membrane $(p v m)$ lined with electron dense layer $(e d l)$ that is thinned out or interrupted at places (double arrowheads) at different levels of the vp and thick ground substance layer $(g s)$ without microtubules but with finer granular material. A few electron dense granules were present in the gs (arrowheads). The gs is juxtaposed with bradyzoite $(b r)$. 
All sarcocysts were mature and they were up to $1758 \mu \mathrm{m}$ long and $145 \mu \mathrm{m}$ wide (Fig. 1c, d). The sarcocysts were septate and centers of some of them were empty or contained degenerated parasites (Fig. 1c). At $\times 400$ magnification, sarcocyst wall appeared to be smooth but few irregular protrusions were visible in some sarcocysts at $\times 1000$ magnification; the sarcocyst wall was up to $1.5 \mu \mathrm{m}$ thick (Fig. 1d). Except for focal infiltrations of mononuclear cells, inflammatory responses were not seen even in heavily infected foxes. There was no evidence of sarcocyst degeneration.

\section{Transmission electron microscopy}

Five sarcocysts (4 from 1 and 1 from another fox) were studied ultrastructurally. All five sarcocysts were structurally similar. The parasitophorous vacuolar membrane (pvm) was folded into villar protrusions (vp) and lined by a $100 \mathrm{~nm}$ thick electron dense layer. The pvm was wavy and the electron dense layer (edl) was thinned out or absent at different levels of the vp (Figs. 2, 3, and 4). The vp were up to $3.0 \mu \mathrm{m}$ long and $0.5 \mu \mathrm{m}$ wide, pleomorphic, some appeared anastomosing and they lacked microtubules (Figs. 3 and 4). The ground substance (gs) was smooth, without any microtubules or granules, and up to $2.5 \mu \mathrm{m}$ thick. The total thickness of the wall including vp and the gs was up to $4.0 \mu \mathrm{m}$. All sarcocysts were mature and contained metrocytes and bradyzoites (Figs. 2, 3, 5, and 6).

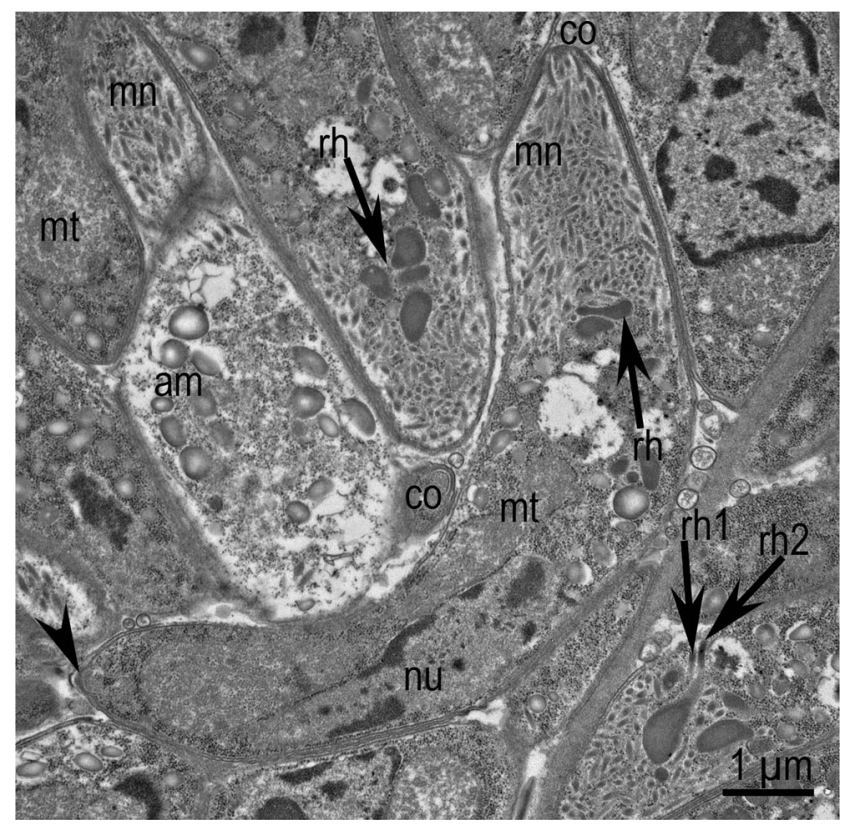

Fig. 6 TEM of bradyzoites in sarcocyst \#1 from the Alaskan Arctic fox. Sections of several bradyzoites. Note a conoid (co), many amylopectin granules $(\mathrm{am})$, numerous micronemes $(\mathrm{mn})$, an elongated nucleus $(\mathrm{nu})$, and an elongated mitochondrion $(m t)$. There are at least two rhoptries $(\mathrm{rh} 1, \mathrm{rh} 2)$ in each bradyzoite with a narrow neck and large blind ends (arrows).



Fig. 5 TEM of sarcocyst \#1 from the Alaskan Arctic fox. Note a group of metrocytes (me) containing several amylopectin granules (arrows), prominent nucleus $(n u)$, and no micronemes nor rhoptries. Also note, host cell $(h c)$, ground substance layer $(g s)$, and villar protrusions $(v p)$ 
The metrocytes were arranged or grouped together (Fig. 5). Metrocytes contained few organelles except that there were several amylopectin (am) granules, which is an unusual finding. In sections examined, metrocytes lacked rhoptries and micromes (Fig. 5). In TEM sections, bradyzoites were curved and difficult to measure. They contained organelles typical of Sarcocystis bradyzoites, including conoid, micronemes, rhoptries, mitochondrion, nucleus, and amylopectin granules (Fig. 6). There were numerous micronemes, arranged haphazardly in the conoidal third of the bradyzoite (Fig. 6). There were at least two rhoptries with a large bulbous blind end. The nucleus was often elongated and located subterminally. The amylopectin granules were scattered in posterior two-third of the bradyzoite. The posterior end of the bradyzoite had a conical thickening (Fig. 6).

\section{Molecular analyses}

Sequences from the $18 \mathrm{~S}$ rDNA, 28S rDNA, ITS-1, and cox1 loci were remarkably similar to previously published S. arctica sequences (Figs. 7 and 8). Both 18S rDNA sequences (1756 bp in length) were $100 \%$ identical to each other
(KY947306; KY947307) as well as seven previously published $S$. arctica sequences derived from Alaskan wolves (KX022100, KX022101, KX022102) and Norwegian Arctic foxes (KF601301, KF601302, KF601303, KF601304). Each $28 \mathrm{~S}$ sequence was identical to published $S$. arctica sequences, but the two differed by a single nucleotide (KY947308; KY947309). No other Sarcocystis species had fewer than 12 nucleotide differences at the 18S rDNA locus or 27 differences at the $28 \mathrm{~S}$ rDNA locus. The ITS-1 had more intraspecific sequence variation with a maximum of five differences between any two sequences, as expected (Gjerde and Josefsen 2015). The ITS-1 sequence derived from one Arctic fox sampled here (KY947310; KY947311) was identical to Norwegian S. arctica sequences (KF601306, KF601307, KF601309, KF601310, and KF601311), while the sequence from the other fox was nearly identical (99.9\% identical) to S. arctica from an Alaskan wolf (Canis lupus, KX022108). There was greater than $98 \%$ identity among all S. arctica sequences (Fig. 7). No other species of Sarcocystis could be reliably aligned at the ITS locus. Cox1 sequences amplified from two different Arctic foxes were identical to each other (KY947304; KY947305) as well as S. arctica from Alaskan
Fig. 7 A neighbor-joining 18S ribosomal DNA gene tree shows strong support for interspecific differences among Sarcocystis species. From alignments of $1756 \mathrm{bp}$, a consensus tree was constructed from 1000 bootstrap replicates based on HKY genetic distances rooted by E. tenella. Sarcocystis arctica $18 \mathrm{~S}$ rDNA sequences isolated from Alaskan Arctic foxes, Alaskan wolves, and Norwegian Arctic foxes were restricted to a single branch with $100 \%$ bootstrap support. The $S$. arctica branch falls within a clade with $100 \%$ bootstrap support which includes $S$. lutrae, S. turdusi, S. speeri, and S. rileyi. The remaining species agreed with previous analyses (Gjerde and Schulze 2014) and are shown for consistency with past analyses. Bootstrap values are only shown for those partitions that appeared in greater than $85 \%$ of replicates
Alaskan wolf

Alaskan Arctic fox

Norwegian fox
KX022101 Sarcocystis arctica KX022102 Sarcocystis arctica KX022100 Sarcocystis arctica KY947306 Sarcocystis arctica-like, Cyst 1 100 KY947307 Sarcocystis arctica-like, Cyst 2 KF601301 Sarcocystis arctica KF601302 Sarcocystis arctica $\bigcirc$ KF601303 Sarcocystis arctica $\bigcirc$ KF601304 Sarcocystis arctica $\bigcirc$ $100{ }_{100}$ GU120092 Sarcocystis rileyi KJ396583 Sarcocystis rileyi KM657769 Sarcocystis lutrae KM657770 Sarcocystis lutrae - KT207459 Sarcocystis speeri JF975681 Sarcocystis turdusi

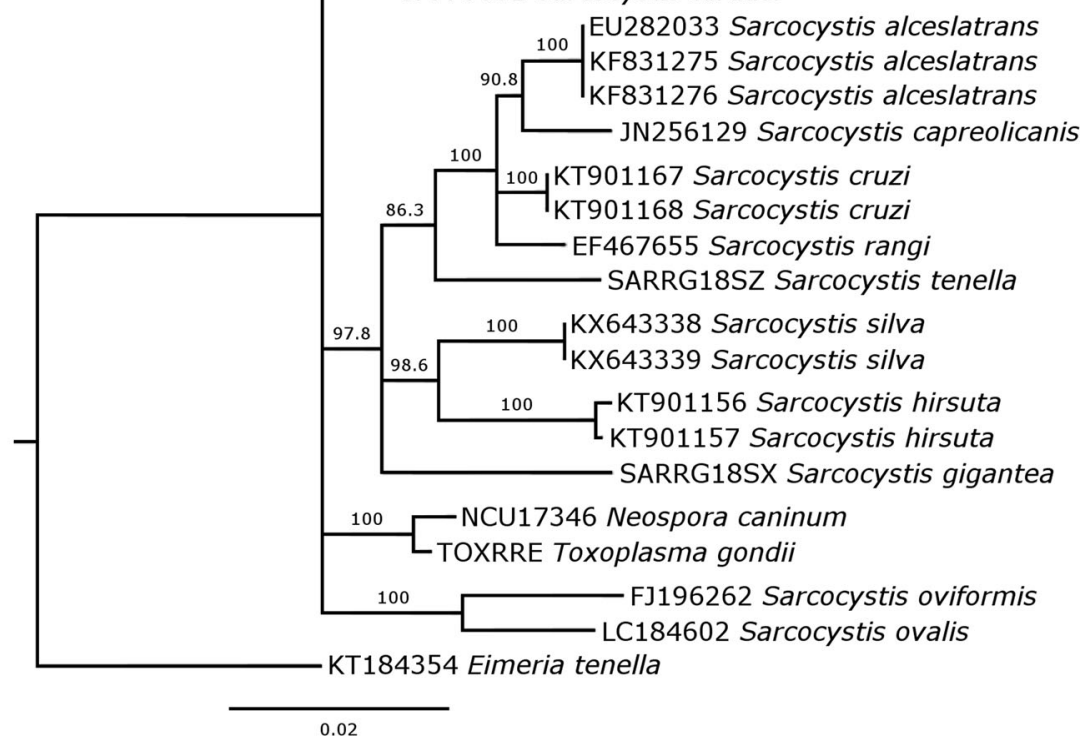


Fig. 8 The mitochondrial cytochrome oxidase I ( $\operatorname{cox} 1)$ gene tree supports the inclusion of Alaskan Arctic fox sarcocysts in the species $S$. arctica. A neighborjoining consensus tree was constructed from 1000 bootstrap replicates based on alignments of 917 bp and HKY genetic distances. The branch encompassing all $S$. arctica cox 1 sequences and sequences from Alaskan Arctic fox sarcocysts had 99.3\% bootstrap support. Similar to the $18 \mathrm{~S}$ rDNA gene tree,

$S$. arctica sequences were in a clade with S. lutrae, S. turdusi, S. speeri, and $S$. rileyi. At the cox 1 locus, the $S$. arctica clade was more closely related to Neospora caninum and Toxoplasma gondii than other Sarcocystis species. The gene tree was rooted with cox1 sequence from Eimeria tenella

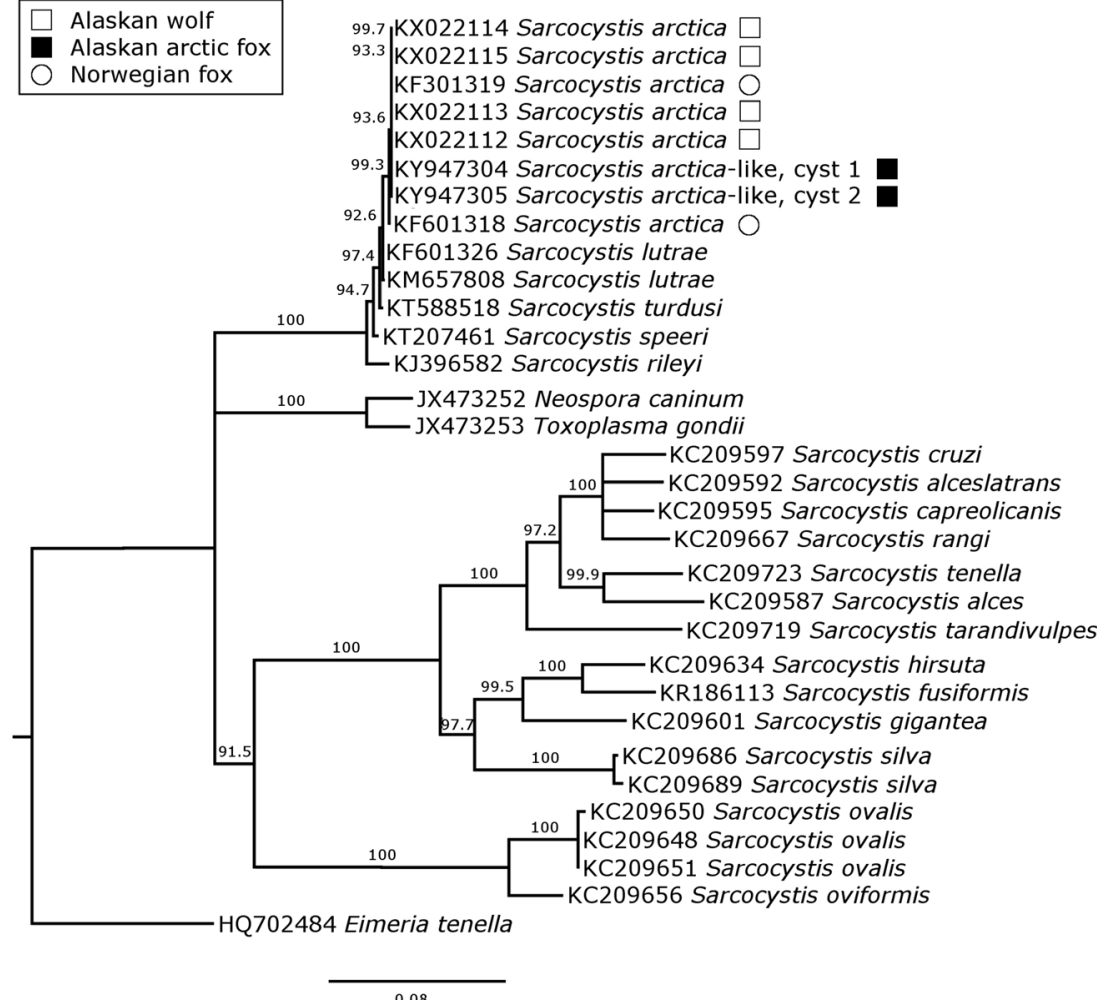

wolf (KX022112; KX022113; KX022114; KX022115) (Calero-Bernal et al. 2016) and Norwegian Arctic fox (KF601319) (Gjerde and Schulze 2014). This shared 99.3\% similarity with $S$. lutrae from Norwegian Eurasian otters (KF601326) (Gjerde and Josefsen 2015) and 98.9\% similarity with $S$. turdusi (KT588518) (Prakas et al. unpublished). Our phylogenetic study denotes that $S$. arctica from Alaskan wolf (Calero-Bernal et al. 2016), S. arctica from Norwegian Arctic foxes (Gjerde and Schulze 2014) and Sarcocystis from Alaskan Arctic foxes (present study) are in the same clade. That conclusion is supported here as neighbor-joining $18 \mathrm{~S}$ rDNA and cox 1 trees show greater than $99 \%$ bootstrap support for a clade encompassing all $S$. arctica sequences, including the sequences derived from sarcocysts present in Arctic fox tongues presented here.

\section{Discussion}

Sarcocysts found in Arctic foxes from Alaska appeared similar to the S. arctica sarcocysts found in two Norwegian Arctic foxes (Gjerde and Schulze 2014). Sarcocysts from one Norwegian fox were previously described morphologically by light microscopy. They were up to $12 \mathrm{~mm}$ long in muscle squashes and had serrations on the sarcocyst wall (Gjerde and Schulze 2014). However, the serrations were not seen in HEstained sections. Sarcocysts were not detected by light microscopy from the second fox. Sarcocysts were characterized molecularly from both foxes.

The tissues examined may contribute to minor differences seen (by light microscopy of sarcocysts) in our report and the previous one from Norway. Here, only tongues were available for examination whereas in the study from Norway, body muscles other than tongue were examined. The length of sarcocysts can vary according to the muscle parasitized; sarcocysts are smaller in size in the myocardium versus skeletal muscle (Dubey et al. 2016). Sarcocysts from the Norwegian Arctic foxes were not described ultrastructurally. We have now added the ultrastructural description of sarcocysts, including the sarcocyst wall, metrocytes, and bradyzoites. Ultrastructurally, the sarcocyst wall of sarcocyst from the Arctic fox most closely resembles "type 9c" sarcocyst wall of Dubey et al. (2016). Molecularly, the S. arctica sarcocysts in Arctic foxes from both continents appear similar. Comparing molecular characteristics, the regions 18S rRNA, 28S rRNA and cox1, which are normally more conserved, shared 100,99.9, and $100 \%$ identity with S. arctica found in Alaskan wolf and 100, 99.9, and 99.3\% identity with $S$. arctica sequences from Norwegian foxes. On the other hand, the ITS-1 locus, which is expected to have more variability, showed a split between the sequences with respect to their similarity to published sequences. One sequence was most similar to $S$. arctica from an Alaskan wolf while the other was identical to $S$. arctica ITS-1 from Norwegian foxes. In general, sarcocysts of $S$. arctica from 
Norway and USA are similar to $S$. arctica-like sarcocysts from the gray wolf from Alaska (Calero-Bernal et al. 2016) and S. caninum sarcocysts from dogs from USA (Dubey et al. 2015). The definitive host/hosts of these sarcocysts are unknown. Many species of carnivores, including birds, bears, and even foxes prey on foxes or scavenge fox carcasses. The molecular characteristics of $S$. arctica from Arctic foxes in Alaska reported here and from Norway by Gjerde and Schulze (2014) should help in the identification of Sarcocystis sporocysts of this species in carnivorous definitive host.

This is the first report of Sarcocystis infection in Alaskan Arctic fox.

Acknowledgements We thank Justin Fry for his help collecting samples in the field. Mention of trade names or commercial products in this publication is solely for the purpose of providing specific information and does not imply recommendation or endorsement by the US Government; US Government is an equal opportunity provider and employer.

\section{References}

Calero-Bernal R, Cerqueira-Cézar CK, Verma SK, Mowery J, Carmena D, Beckmen K, Dubey JP (2016) Sarcocystis arctica (Apicomplexa: Sarcocystidae): ultrastructural description and its new host record, the Alaskan wolf (Canis lupus). Parasitol Res 115:2893-2897. doi: 10.1007/s00436-016-5067-5

Dubey JP (2010) Toxoplasmosis of animals and humans, 2nd edn. CRC Press, Boca Raton
Dubey JP, Sykes JE, Shelton GD, Sharp N, Verma SK, Calero-Bernal R, Viviano J, Sundar N, Khan A, Grigg ME (2015) Sarcocystis caninum and Sarcocystis svanai n. spp. (Apicomplexa: Sarcocystidae) associated with severe myositis and hepatitis in the domestic dog (Canis familiaris). J Eukaryot Microbiol 62:307-317. doi:10.1111/jeu.12182

Dubey JP, Calero-Bernal R, Rosenthal BM, Speer CA, Fayer R (2016) Sarcocystosis of animals and humans, 2nd edn. CRC Press, Boca Raton

Gjerde B (2013) Phylogenetic relationships among Sarcocystis species in cervids, cattle and sheep inferred from the mitochondrial cytochrome c oxidase subunit I gene. Int J Parasitol 43:579-591. doi: 10.1016/j.ijpara.2013.02.004

Gjerde B (2014) Morphological and molecular characteristics of four Sarcocystis spp. in Canadian moose (Alces alces), including Sarcocystis taeniata n. sp. Parasitol Res 113:1591-1604. doi:10. 1007/s00436-014-3806-z

Gjerde B, Josefsen TD (2015) Molecular characterisation of Sarcocystis lutrae n. sp. and Toxoplasma gondii from the musculature of two Eurasian otters (Lutra lutra) in Norway. Parasitol Res 114:873-886. doi:10.1007/s00436-014-4251-8

Gjerde B, Schulze J (2014) Muscular sarcocystosis in two Arctic foxes (Vulpes lagopus) due to Sarcocystis arctica $\mathrm{n}$. sp.: sarcocyst morphology, molecular characteristics and phylogeny. Parasitol Res 113:811-821. doi:10.1007/s00436-013-3711-x

Scioscia NP, Olmos L, Gorosábel A, Bernad L, Pedrana J, Hecker YP, Gual I, Gos ML, Denegri GM, Moore DP, Moré G (2017) Pampas fox (Lycalopex gymnocercus) new intermediate host of Sarcocystis svanai (Apicomplexa: Sarcocystidae). Parasitol Int 66:214-218. doi:10.1016/j.print.2017.01.021

Trupkiewicz JG, Calero-Bernal R, Verma SK, Mowery J, Davison S, Habecker P, Georoff TA, Ialeggio DM, Dubey JP (2016) Acute, fatal Sarcocystis calchasi-associated hepatitis in roller pigeons (Columba livia f. dom.) at Philadelphia zoo. Vet Parasitol 216:52-58. doi:10. 1016/j.vetpar.2015.11.008 